\title{
The coherent sound field separation method combining compressive sensing
}

\author{
Chen Hantao ${ }^{1}$, Guo Wenyong ${ }^{2}$, Zhang Hongyu ${ }^{3}$ \\ College of Power Engineering, Naval University of Engineering, Wuhan, China \\ ${ }^{1}$ Corresponding author

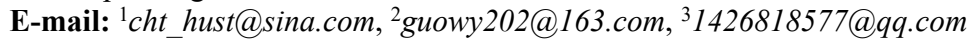

Received 26 April 2018; accepted 7 May 2018

DOI https://doi.org/10.21595/vp.2018.19957

Check for updates

Copyright $(0) 2018$ Chen Hantao, et al. This is an open access article distributed under the Creative Commons Attribution License, which permits unrestricted use, distribution, and reproduction in any medium, provided the original work is properly cited.

\begin{abstract}
When multiple coherent sound sources are distributed on the same side of the holographic surface, the conventional method must use known information such as sound source distribution and geometry. In view of this situation, a method of coherent sound field separation combined with compressive sensing is proposed. The method is based on the plane equivalent source near-field acoustic holography method and the orthogonal matching pursuit algorithm of compressive sensing technology. Get the distribution of the virtual sound source on the plane, and then the virtual sound sources are grouped and selected. Finally, the distribution of sound pressure in the near-field plane after separation of the sound field is calculated using the conduction matrix. The method does not require other prior knowledge. The simulation results show that this method can be used as an effective complementary method for coherent sound field separation based on single-sided measurement.
\end{abstract}

Keywords: near-field acoustic holography, coherent sound field, compressed sensing, equivalent source method.

\section{Introduction}

The existing acoustic imaging technology is mainly implemented by near-field acoustical holography (NAH) $[1,2]$. When there are multiple coherent sources in the sound field and they are on the same side of the holographic surface, the holographic surface measurement results in a composite sound field produced by each coherent source. At this time, the sound pressure distribution of a single sound source and its contribution in the sound field cannot be obtained by the conventional near-field acoustic holography methods.

The NAH-based sound field separation technology research began in the 1980s. This technology is divided into single-side sound field separation technology and double-sided sound field separation technology. Double -side sound field separation methods needed to measure sound pressure data on two planes. These methods were less efficient, and the effect of the double-sided separation method was closely related to the distance between double measurement planes. At present, there is no clear basis for the selection of measurement planes [3].

Many scholars have also conducted extensive research on sound field separation technology based on single-sided measurement. Part of the field decomposition techniques proposed by Hallman et al. [4] could be used to analyze the multi-source sound field on the same side of the holographic surface as the coherent sound source. However, the position of the sound source needed to be known in advance and the reference microphone was arranged to obtain the signal related to the sound source. Wu et al. [5] proposed a hybrid near-field acoustic holography technique. However, there was still needing to add an auxiliary surface in the selection of the optimal cut-off order of spherical wave. Two measurements were needed in the actual measurement. Zhang Yongbin et al. [6] proposed a hybrid source-based coherent sound source separation method, but it was necessary to place an orthogonal spherical wave source at each sound source.

For coherent sound sources on the same side of the holographic plane, a method of coherent 
sound field separation combined with compressed sensing technology is proposed in this paper. The method only needs a single-sided measurement, and it does not require other prior knowledge. The simulation results show that this method can be used as an effective complementary method for coherent sound field separation based on single-sided measurement.

\section{Theoretical model and algorithm implementation}

\subsection{Plane equivalent source method near-field acoustic radiation calculation model}

Setting the virtual sound sources on a plane, which has better convenience and versatility. As shown in Fig. 1, $S_{v}$ represents the virtual sound source plane, $S_{s}$ represents the sound source plane, $S_{f}$ represents a plane in the sound field, $S_{h}$ represents the holographic surface, $n$ represents the normal direction of the sound source outside the plane, $P_{h}$ is the sound pressure distribution on the holographic surface, and $P_{S}$ is the sound source plane Sound pressure distribution, $Q_{v}$ is the sound intensity of the virtual sound source.

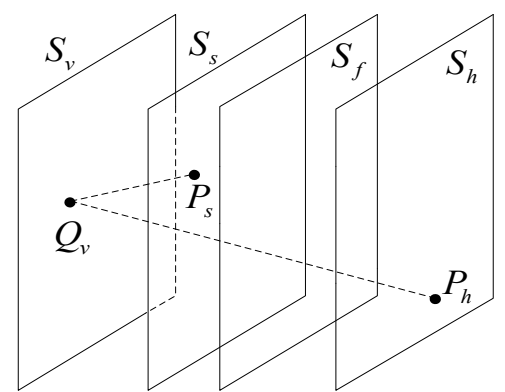

Fig. 1. A schematic diagram of Plane ESM

The sound pressure distribution on the holographic surface can be represented by the following integral equation:

$p_{h}(\mathbf{r})=\int_{S_{v}} i \rho c k q_{v}\left(\mathbf{r}_{0}\right) g\left(\mathbf{r}, \mathbf{r}_{0}\right) d S_{v}$,

where $g\left(\mathbf{r}, \mathbf{r}_{0}\right)$ is the free space Green's function from the virtual point source to the point which is on the holographic surface, $q_{v}\left(\mathbf{r}_{0}\right)$ is the virtual point sound source intensity, $\rho$ is the medium density, $c$ is the sound speed, and $k$ is the wave number. The sound pressure on the actual sound source surface can be expressed in the following form after being discretized:

$P_{s}=G_{s v} Q_{v}$,

where $P_{S}$ is the sound source surface acoustic pressure column vector, $Q_{v}$ is the source strong column vector of the equivalent source sequence, and $G_{s v}$ is the sound pressure matching matrix between the equivalent source sequence and the sound source surface:

$G_{s v}=i \rho c k g\left(r_{s}, r_{v}\right)$

After the sound pressure on the holographic surface is discretized, it can be expressed as:

$P_{h}=G_{h v} Q_{v}$.

$P_{h}$ is the sound pressure column vector on the holographic surface, $Q_{v}$ is the source strong 
column vector of the equivalent source sequence, and $G_{h v}$ is the sound pressure matching matrix between the equivalent source sequence and the holographic surface:

$G_{h v}=i \rho c k g\left(r_{h}, r_{v}\right)$.

The inversion formula for the virtual source strength is:

$Q_{v}=G_{h v}^{+} P_{h}$.

Further, the sound pressure of the actual sound source surface can be expressed as:

$P_{s}=G_{s v} G_{h v}^{+} P_{h}$.

The sound pressure transfer matrix from the sound source surface to the holographic surface is expressed as:

$L=G_{v S} G_{v h}^{+}$

Get the sound pressure $P_{S}$ on the sound source surface:

$P_{s}=L P_{h}$.

Knowing $L$ and $P_{h}$, the sound pressure distribution on the sound source plane can be reconstructed. If know the sound pressure transfer matrix $L$ from the sound source plane $S_{S}$ to a certain plane $S_{f}$ in the sound field and the sound pressure $P_{h}$ on the holographic plane, the sound pressure distribution on any plane $S_{f}$ in the sound field can also be reconstructed.

\subsection{Sparse representation model of acoustic pressure array signals}

As shown in Fig. 2, on the holographic plane $S_{h}$, there is a regular array of $M$-element microphones. The sound source points are sparsely distributed on the sound source surface $S_{s}$. The sound source surface $S_{S}$ can be evenly divided into grid points so that the sound source points can be sparsely represented on the sound source surface [7].

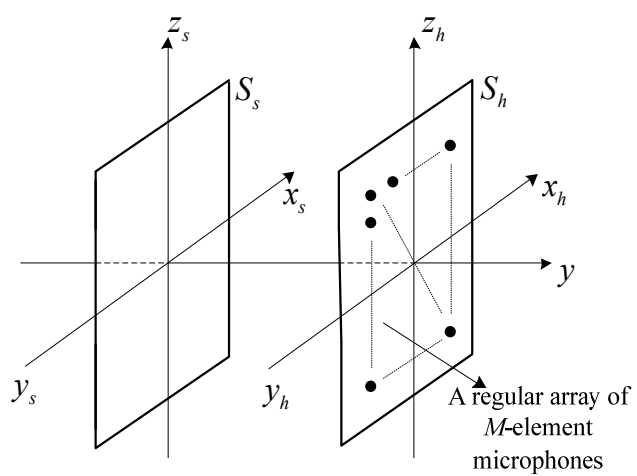

Fig. 2. Microphone array signal sampling model

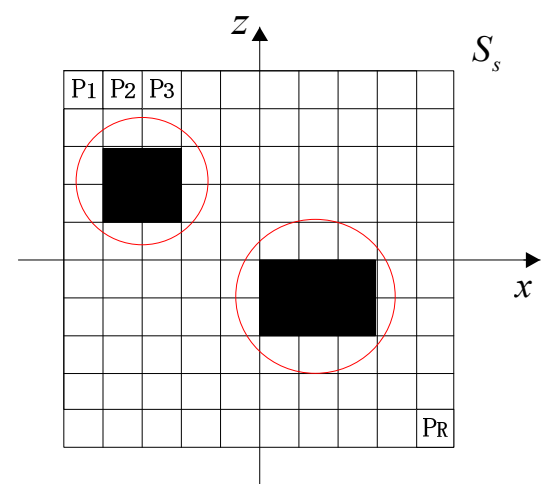

Fig. 3. Sound source surface sparse representation

As shown in Fig. 3, the sound source surface $S_{S}$ is divided into $R$ areas. A solid area indicates that there is a sound source at that location, and a hollow area indicates that there is no sound source. A single sound source is often a small area element, which can be viewed as a collection of several point sources. The sound pressure signals received by the regularly distributed $M$-element microphone array is sparsely represented as: 
$y=A x+\theta$.

In the formula, $y$ is an $M \times 1$ dimensional matrix, which represents the signal received by the microphone array. $A$ is an $M \times R$ dimensional matrix that represents the sensing matrix. $x$ is an $R \times 1$ dimensional matrix and represents a sound source signal containing position information, of which there are only $N(N \ll R)$ non-zero values. $\theta$ is the received noise signal.

\subsection{A coherent sound field separation method combined with compression sensing}

In the planar equivalent source near-field acoustic radiation model, the virtual sound source surface $S_{v}$ is discretized. The virtual sound source points are sparsely distributed on $S_{v}$. The transfer matrix in the plane ESM is directly used as a sensing matrix, and the matrix conforms to the Restricted Isometry Property (RIP) [8]. Firstly, the position of the virtual sound sources on the virtual sound source surface is obtained by orthogonal matching pursuit (OMP) algorithm, and then the near-field plane sound pressure distribution is reconstructed in combination with the known transfer matrix. In the plane rectangular coordinate system, the virtual sound source plane may be discretized into $I \times I$ uniform squares, and the microphones on the holographic surface may also be evenly spaced to facilitate calculation. The sound pressure transfer matrix from the sound source plane $S_{s}$ to a plane $S_{f}$ in the sound field can be expressed as:

$L=\mathbf{G}_{v f} \mathbf{G}_{v h}^{+}$.

This is the sensing matrix, $G_{v f}$ is the sound pressure matching matrix between the equivalent source sequence and a plane in the sound field:

$G_{v f}=i \rho c k g\left(r, r_{v}\right)$.

Substituting Eq. (11) into Eq. (10) yields:

$y=L x+\theta$

The presence of a noise signal will affect the accuracy of sound source localization, where $\theta$ is the noise signal, which is directly recorded by the array, so Eq. (13) can be written as:

$y=L x$.

According to the flow of the OMP reconstruction algorithm $[9,10]$, it is necessary to input the sensing matrix $L$, the observation vector $y$, and the signal sparsity $K$. In practice, a single virtual sound source is a small area element, which can be regarded as a collection of several point sources, so the input sparsity K can be approximated. Referring to the example of the OMP reconstruction algorithm given by Tropp [10], after knowing the relationship between the sampled value $M$ and the reconstruction success rate and the relationship between the signal sparsity $K$ and the reconstruction success rate, the sparsity $K$ can be determined.

After finding the sparse distribution $x^{\prime}$ of the virtual sound sources, the virtual sound sources are grouped, and those grouped together are grouped into the same group. As shown in Fig. 3, the same group is in the red circle. Assuming there are two coherent sound sources, the virtual sources are divided into two groups, one of which is selected to obtain a sparse distribution $x^{\prime \prime}$.

The transfer matrix is known to be calculated in combination with $x^{\prime}$ and $x^{\prime \prime}$ :

$y^{\prime} d=L d x^{\prime}$,

$y^{\prime \prime} d=L d x^{\prime \prime}$. 
Subtract the two equations to obtain the separated sound field on any near-field plane $S_{f}$ that is a distance $d$ from the actual sound source plane:

$y_{d}=y_{d}^{\prime}-y_{d}^{\prime \prime}=L_{d} x^{\prime}-L_{d} x^{\prime \prime}$.

\section{Simulation analysis}

\subsection{Discs vibration model}

Sound radiation model shown in Fig. 4. Both discs have a radius of $0.05 \mathrm{~m}$ and a thickness of $0.008 \mathrm{~m}$. Both materials are alloy structural steels. The central axis of the two discs is parallel to the $y$-axis, and the two discs are equally distributed along the $y$-axis. The coordinates of the center of the discs distributed are $(0.2,0,0.2)$ and $(-0.2,0,-0.2)$. A $10 \mathrm{~N}$ excitation force is applied in the normal direction at the center of both discs at a frequency of $500 \mathrm{~Hz}$.

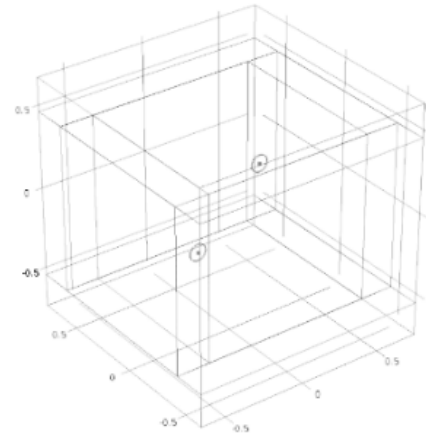

Fig. 4. Sound field three-dimensional model

\subsection{Sound field holographic simulation imaging analysis}

Fig. 5 shows the sound pressure distribution of an ideal sound field separated on a plane $0.1 \mathrm{~m}$ from the side of the discs. From the side of the disc $0.2 \mathrm{~m}$ on the plane, the sound pressure distribution shown in Fig. 6.

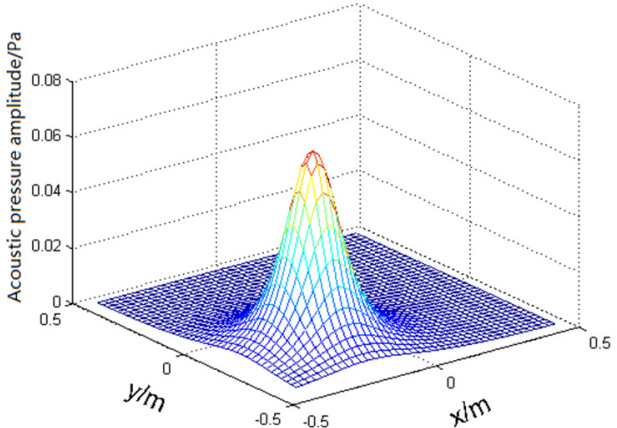

a)

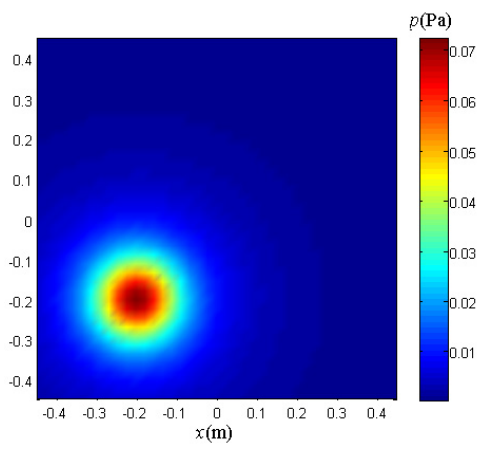

b)

Fig. 5. Sound pressure distribution of an ideal sound field separated

A $11 \times 11$ array microphones are used to sample the sound pressure shown in Fig. 6 . Divide the virtual sound source surface into $16 \times 16$ grids. At this time, according to the example of Tropp, when the sparsity $K$ is 22 , there is good reconstruction success rate. When reconstructing the sound pressure distribution of the separated sound field in the plane of $0.1 \mathrm{~m}$ from the sound source plane, the plane is divided into $41 \times 41$ grids for sound pressure reconstruction. 
Use the method proposed in this paper to separate the composite sound pressure on the holographic surface. Fig. 7 shows the virtual sound sources distribution. Only the sound sources in the lower left red circle participate in the sound pressure reconstruction. The result of the calculation is shown in Fig. 8.

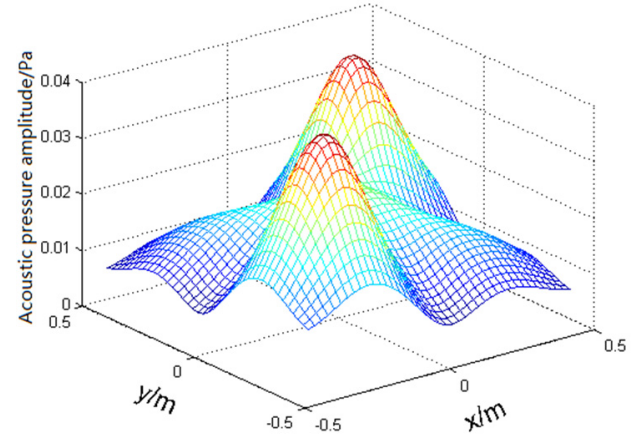

a)

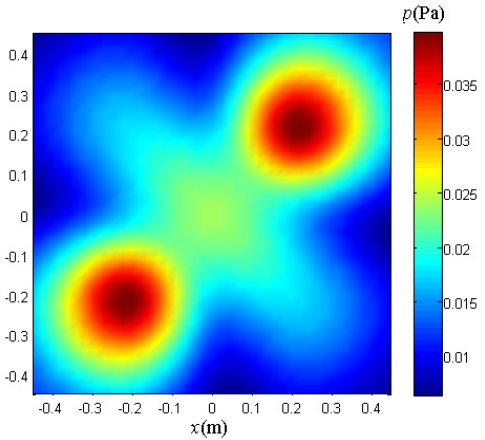

b)

Fig. 6. Plane sound pressure distribution at $0.2 \mathrm{~m}$

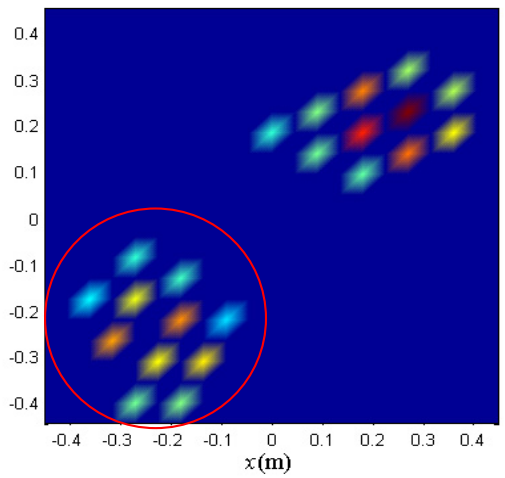

Fig. 7. Virtual sources distribution

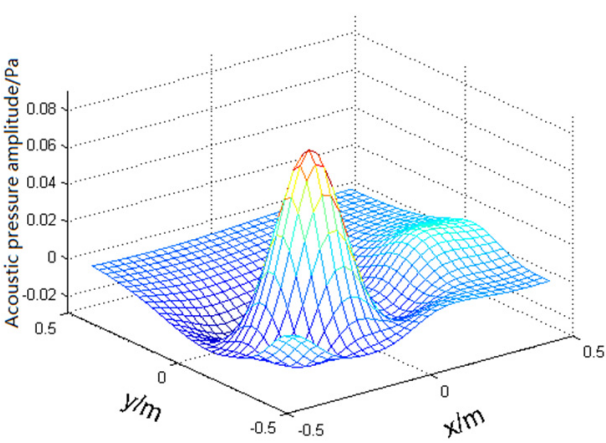

a)

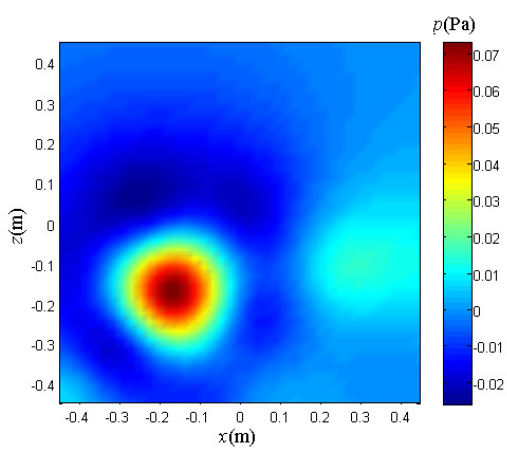

b)

Fig. 8. Sound field separation result graph

\subsection{Results and discussion}

The composite sound pressure shown in Fig. 6 is separated according to the method proposed in this paper. The results obtained are shown in Fig. 8. The coherent sound field is separated using the method proposed in this paper. The results obtained are good, and the sound source location is accurate, but there is a slight error in the local sound pressure value. 
As shown in Fig. 9, a scatter plot is made for the difference between the calculated value and the theoretical value of the separated sound field, which is $41 \times 41$ points in total. The errors are all around zero. There are only little local errors, which is consistent with Fig. 8.

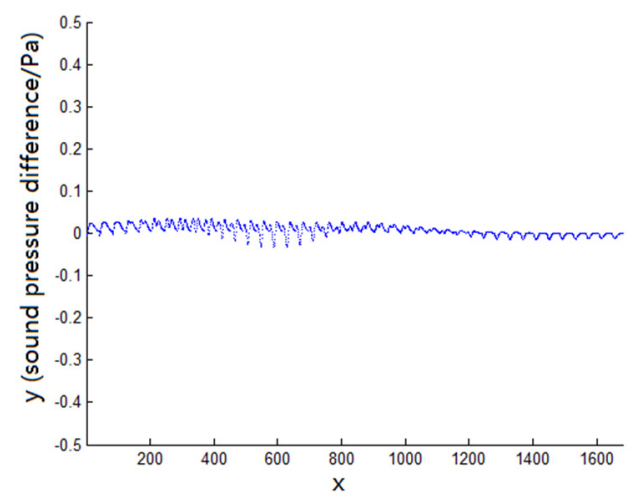

Fig. 9. Sound pressure differences scatter plot

\section{Conclusions}

1) This paper proposes a method for the separation of multi-source coherent sound field based on single-side measurement without prior knowledge, which provides a powerful method for the analysis of coherent sound field.

2) The separation of coherent sound field composed of two vibrating discs is simulated and the separation errors are small. The obtained results well prove the feasibility and effectiveness of the method.

\section{References}

[1] Williams E. G., Maynard J. D., Skudrzyk E. Sound source reconstructions using a microphone array. Journal of the Acoustical Society of America, Vol. 68, Issue 1, 1980, p. 340-344.

[2] Williams E. G., Maynard J. D. Holographic imaging without the wavelength resolution limit. Physical Review Letters, Vol. 45, Issue 7, 1980, p. 554-557.

[3] Yu Fei, Chenjian, Bi Chuanxing, et al. Experimental investigation of sound field separation technique with double holographic planes. Acta Acustica, Vol. 30, Issue 5, 2005, p. 452-456, (in Chinese).

[4] Hallman D., Bolton J. S. Multi-reference nearfield acoustical holography. Proceedings of INTER-NOISE, Toronto, Canada, 1992, p. 1165-1170.

[5] Wu S. F. Hybrid near-field acoustic holography. Journal of the Acoustics Society of America, Vol. 115, Issue 1, 2004, p. 207-217.

[6] Zhang Yongbin, Bi Chuanxing, Chen Jian, et al. Separation method of coherent sources based on hybrid holographic algorithm. Journal of Mechanical Engineering, Vol. 43, Issue 9, 2007, p. 173-178, (in Chinese).

[7] Shi Jie, Yang Desen, Shi Shengguo, et al. Compressive focused beamforming based on vector sensor array. Acta Physica Sinica, Vol. 65, Issue 2, 2016, p. 190-200.

[8] Zhang B., Cheng X., Zhang N., et al. Sparse target counting and localization in sensor networks based on compressive sensing. Proceedings, IEEE Infocom, Vol. 2, Issue 3, 2011, p. 2255-2263.

[9] Ning Fangli, Wei Jingang, Liu Yong, et al. Study on sound sources localization using compressive sensing. Journal of Mechanical Engineering, Vol. 52, Issue 19, 2016, p. 42-52, (in Chinese).

[10] Tropp J. A., Gilbert A. C. Signal recovery from random measurements via orthogonal matching pursuit. IEEE Transactions on Information Theory, Vol. 53, Issue 12, 2007, p. 4655-4666. 